\title{
Discussion on Leakage Protection in Distribution Network
}

\author{
Zhang Xiaoshu ${ }^{1}$, Cao Huiyan ${ }^{1}$, Zhao Chuanzong ${ }^{1}$, Xu Mei ${ }^{1}$, Li Tianci ${ }^{1}$, Ye Dewu ${ }^{1}$, Zhang \\ Jingyi $^{2}$, Zhu Jiahui ${ }^{1}$, Yue Yangzhuo ${ }^{1}$, Song Yuanlin ${ }^{1}$ \\ ${ }^{1}$ Fushun Power Supply Company, Liaoning Electric Power Company Limited, State Grid, China \\ ${ }^{2}$ Laoning Bidding Company Limited, State Grid, China
}

Keywords: Leakage protection, Low voltage distribution network, The power safety

\begin{abstract}
Although the voltage level of distribution network is low, the variety of power equipment is complicated, the users are uneven, and there is a greater risk of electric shock. The problem of leakage in distribution line are discussed. First of all, the leakage protection is systematically analyzed, then the correct use of leakage protection in distribution network is studied. Finally, the common problems of leakage protection are expounded from the human level and material level. The solution suggestion is given. The research of leakage protection is of great significance to promote the application of leakage protection. The purpose is to make the staff understand the correct use of leakage protection, which has certain promotion value.
\end{abstract}

\section{Introduction}

With the large increase of the low voltage load of the distribution network, on the one hand, it requires the distribution network transformation to follow up in time, on the other hand, the security level of the distribution network must be improved. There are defects in the design of low-voltage distribution network. Because the long line was wet, the capacitance of current is large. The insulation of the wire line is aging and breaking. As a result of the above reasons, the phenomenon of leakage current exceeding the standard occurs frequently. In order not to interrupt the power supply, some users will remove the leakage protector under special circumstances. When the leakage grounding occurs, the protection will not act, resulting in equipment damage or personal injury accident. Because the leakage current exceeds the action value, the user who has not removed the leakage protection device had a blackout accident caused by the protection action.

The leakage protection device works on the basis of leakage current detection, which is an effective safeguard measure for electric power at present. Therefore, it is of great significance to find the leakage position quickly and accurately, and deal with it in time.

\section{Steps to find leakage}

Usually, the method to find leakage fault can be divided into four steps.

The first step is to determine whether the line is really leaking. At this point, the insulation resistance of the line can be measured by the Megameter table or an ammeter is connected to the total knife gate, all the switches are connected and all the bulbs are removed. If the ammeter needle wobbles, it indicates that the line is leaking.

The second step is to judge whether the leakage between phase line and zero line or between phase line and earth line or both. The method of judging is to cut off the zero line. If the indicator of ammeter was no changed, the leakage would be between the phase line and the earth. If the indicator of ammeter was zero, the leakage would be between the phase line and the zero line. If the indicator of ammeter was small but not zero, the leakage would be both of them.

The third step is to determine the leakage range. Remove the shunt fuse or pull the knife brake. If the indicator of ammeter was unchanged, the leakage would be bus. If the indicator of ammeter was zero, the leakage would be shunt. If the indicator of ammeter was small but not zero, it indicates that the bus and shunt are leaking.

The fourth step is to find the leakage point. After the above inspection, then pull the switch of the 
line lamps and lanterns in turn. When a switch is opened, if the indicator of ammeter returned to zero, the leakage would be the branch line. If the indicator of ammeter becomed smaller, there would be other leakage on the branch line. If all switches of lamps and lanterns have been pulled apart, the leakage would be the main line.

Narrow the scope of the search in turn, you can find out the point of leakage. In order to detect the leakage fault in time, the line should be checked regularly and its insulation resistance should be measured. If the resistance value was smaller, the fault point would be immediately identified and eliminated.

\section{Leakage Protection}

\subsection{The basic structures of leakage protection}

Functionally, the leakage protection device consists of four parts: YA is the electromagnetic release, TAN is the zero sequence current transformer, SB is the switch test button, $\mathrm{R}$ is the resistance, the action principle of leakage protection switch is shown in figure 1

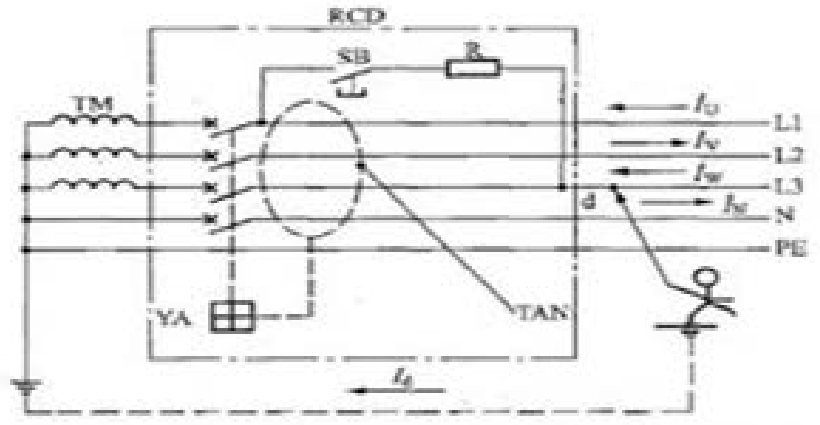

Fig.1 the action principle of leakage protection switch

TM- power transformer; SB- Switch brake testing button; R- resistance

RCD- residual current action protection; YA- Electromagnetic release

TAN- The zero sequence current transformer; $\mathrm{I}_{\mathrm{d}}$ - fault current

When there is no leakage in the network, the three-phase current is balanced, the current through zero-sequence current transformer is zero, and the release does not work. When there is a large leakage current in the distribution network, the zero-sequence current transformer quickly detects and amplifies the signal processing link, and then compared with the safety threshold. If the threshold was larger than the threshold value, an instruction would be generated to make the tripping coil action cut off circuit, so as to achieve the purpose of personal protection.

\subsection{The available range of leakage protection}

Leakage protection acts as a protection against electric shock in both direct and indirect contact protections. The leakage protection can be applied under three situations: The first situation is the bad environment, such as moisture or corrosion. Secondly, leakage protection can be applied to the handheld device, construction equipment, water equipment, medical equipment, etc. Thirdly, leakage protection can be applied to the socket circuit

\subsection{The common leakage protection}

The common leakage protection as shown in figure 2 . 


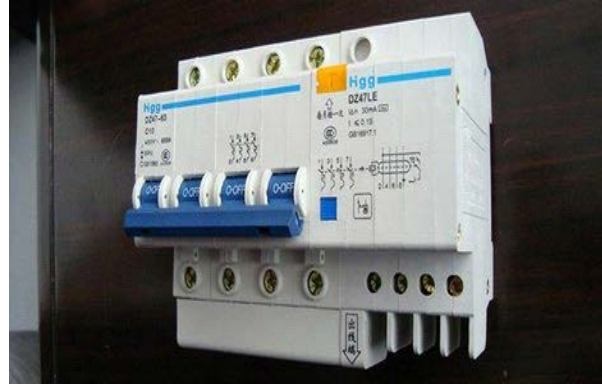

Fig.2 The common leakage protection

\subsection{The classification of the leakage protection}

1) According to the working principle, it can be divided into three types: current type, AC pulse type, voltage action type (tend to be eliminated).

2) According to the adopted components are divided into: electromagnetic type; electronic type.

3) According to the structure, it can be divided into: leakage switch (pointing to leakage signal detection, tripping, tripping mechanism, etc.) assembled in an insulating shell; leakage relay (also called combination type); leakage protection socket.

4) According to the leakage action current, it can be divided into three types: high sensitivity type (Tripping at $6 \mathrm{~mA}$ to $30 \mathrm{~mA}$ ), medium sensitivity type (Tripping at $30 \mathrm{~mA}$ to $1000 \mathrm{~mA}$ ) and low sensitivity type ( $>1000 \mathrm{~mA})$, mainly used to prevent fire and monitor single-phase grounding fault.

5) According to the action time: instantaneous type ( $t \leq 0.2 \mathrm{~s})$; delay type $(0.2 \mathrm{~s}$ can be delayed); reverse time type (the larger the fault current of the protected equipment, the smaller the action delay)

\section{Correct Use of Leakage Protection in Distribution Network}

\subsection{Follow the principle of hierarchical protection}

The leakage protection can not expand the fault while preventing the electric shock. The configuration of the device should follow the principle of graded protection (generally 2 to 4 grades), as shown in figure 3. The operating value and time value of each level should be scientifically coordinated. On the basis of terminal protection, the power supply end should select the low sensitive current value.

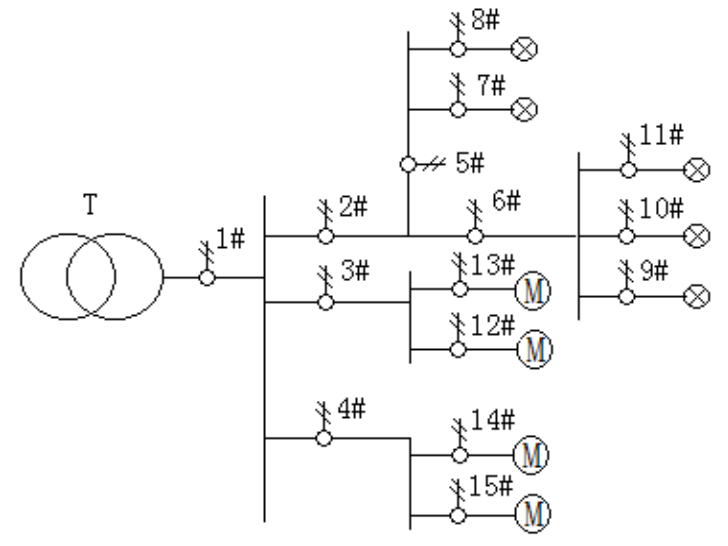

Fig.3 Levels protection of installation position

Note: 1\#-Primary protection (General line protection); 2\#, 3\#, 4\#-Secondary protection (General protection); $7 \#$ to $15 \#-$ Level three protection (final protection); 5\#, 6\# -Level four protection (intermediate protection)

a) When a low voltage line is interrupted on the ground or connected to a telephone line or a low voltage line hits a tree, the primary protection and secondary protection action jump to avoid an electric shock accident. At the same time, when the final stage (Level three) protection has a fault, it can play the role of backup protection. 
b) Final protection (Level three protection) is the leakage switch, which is installed every household, which are required to install in the mobile and hand-held appliances of TT system.

c) If the final protection failure (for example 9\# protection failure), then intermediate protection action tripping (for example 6\# protection).

\subsection{Correct selection and correct connection}

It is often densely staffed on the line terminal, so the leakage protection should be of high sensitivity type, especially for the producing departments working inside the metal container, the operating current should not exceed $15 \mathrm{~mA}$. For the leakage protection which mainly protects against fire, the rated operating current can be relaxed appropriately $(60 \sim 100 \mathrm{~mA})$, and the delay is slightly longer. If the power supply load is very important, the leakage protection device should be alarm first, not immediately removed. If the leakage protection is used to detect the single-phase grounding, the device with delay should be used.

The low-voltage distribution system can be divided into TT system, TN system ${ }^{[3]}$,etc. TN system can be subdivided into TN-S, TN-C, TN-C-S and so on. when the line of leakage protection is connecting, the differential connection should be made in combination with the network type:

In TT and TN-S systems, the leakage protection can protect the terminal protection as well as the whole network, but the neutral line of TN-S system must cross the zero-sequence CT together with the phase lines.

Because the PEN line of TN-C system is not only ground protection but also neutral line, it can not cross its zero sequence CT, although it is connected with RCD. Otherwise, when the grounding fault occurs, the electromagnetic field of the phase line and the PEN line will cancel each other and the fault current will not be detected by RCD. There are two methods to solve it. The first one is to separate the $\mathrm{N}$ line from the PE line, and turn it into a TN-C-S system. The second method is to add the independent protective grounding wires and turn it into a local T-T system.

\section{Common Problems and Solutions of Electrical Leakage Protection}

\subsection{Artificial problem}

(1) With the upgrading of rural power network, the distribution network has been greatly improved. Many users mistakenly believe that the electricity safety has been guaranteed, and there is no need to install the leakage protection device again.

(2) Undeniably, the action threshold of leakage protection does not necessarily cause harm to people. In this way, leakage protection often operates when leakage occurs but is not very serious. At this point, some users will remove the leakage protector to avoid frequent power outages.

(3) Power supply station has not established effective detection, maintenance, publicity and other systems on leakage protection, which is a management vacuum.

\subsection{Equipment problem}

(1) The first problem is the equipment quality problem. Leakage protection devices need to go through the national 3C proof. In rural areas, people lack the ability to distinguish fake and inferior products, it will cause problems such as slippage of actual operating devices and so on.

(2) Connection problem or setting problem. The first one is the protection connection is not in agreement with the network form. The second one is the connection line is loose. The third one is the setting number is bigger or smaller.

(3) Harmonic effect. The extensive use of electronic equipment will cause waveform distortion, which makes the zero-sequence CT of the RCD unable to recognize DC component, which thus is insensitive to leakage fault.

\subsection{Solution to the problem}

Firstly, the responsibility for safety management should be affirmed. It is beneficial to consciously enhance the awareness of leakage protection to the power supply company, users, 
agricultural power workers and other parties, thereby improving the level of safe use of electricity.

Secondly, to improve the network conditions, mainly to improve the unbalanced impedance of power supply.

Finally, the planning, design, procurement, testing, installation, operation and maintenance, emergency and other supporting systems should be established, and seriously implemented.

\section{Conclusions}

Leakage protection is very important to ensure the safety of distribution network. The action value and time value of all levels of protection should be scientifically coordinated. At the same time, the terminal protection should be taken as the basis, and the low sensitivity current value should be selected at the power side in order to avoid the leakage electric shock accident, it is necessary to look for the deficiency of the current leakage protection from the aspects of human and equipment, which may improve the effectiveness of the leakage protection.

\section{References}

[1] Zhao Honghai. Discussion on improving the way of leakage protection in rural low voltage power grid China Electric Power Press [J]. 2002,19(3):132-135.

[2] Li Shibo, Daily Fault Analysis and solution of leakage Protector[J].2007.

[3] Yang Shaofeng, Shi Yubo. A brief discussion on the way of safe Operation Management of Electric Power Dispatch,2011.

[4] Yang Mingfang, Wang Kunlun. Safety Management of Electric Power Operation [J], Economic Forum, 2011. 